\title{
Predictors of treatment failures of Plasmodium falciparum malaria in Vietnam: a 4-year single- center retrospective study
}

\section{Minh Cuong Duong}

University of New South Wales - Kensington Campus: University of New South Wales

Oanh Kieu Nguyet Pham

Hospital for Tropical Diseases

Phong Thanh Nguyen

Hospital for Tropical Diseases

Chau Van Vinh Nguyen

Hospital for Tropical Diseases

Phu Hoan Nguyen ( $\nabla$ Nguyenhoanphu19857@gmail.com )

Vietnam National University Ho Chi Minh City https://orcid.org/0000-0003-2875-1685

\section{Research}

Keywords: Plasmodium falciparum, severe malaria, early treatment failure, late treatment failure, Vietnam

Posted Date: December 16th, 2020

DOI: https://doi.org/10.21203/rs.3.rs-126234/v1

License: (c) (1) This work is licensed under a Creative Commons Attribution 4.0 International License. Read Full License 


\section{Abstract}

\section{Background}

Drug-resistant falciparum malaria is an increasing public health burden. We examined the magnitude of Plasmodium falciparum infection and the patterns and predictors of treatment failure in Vietnam.

\section{Methods}

Medical records of all 443 patients with malaria infection admitted to the Hospital for Tropical Diseases between January 2015 and December 2018 were used to extract information on demographics, risk factors, symptoms, laboratory tests, treatment, and outcomes.

\section{Results}

More than half $(59.8 \%, \mathrm{Cl} 55.1 \%-64.4 \%)$ of patient acquired $P$. falciparum infection of whom $21.9 \%$ (CI 17.1\%-27.4\%) had severe malaria, while 7.2\% (Cl 4.6\%-10.9\%) and 19.2\% (Cl 14.7\%-24.5\%) developed early treatment failure (ETF) and late treatment failure (LTF) respectively. ETF was $4.7 \%$ among patients referred from Binh Phuoc province and Central Highland, $12.9 \%$ from other areas in Vietnam, and 6.9\% from Africa. LTF was $16.2 \%$ among patients from Binh Phuoc province and Central Highland, $22.6 \%$ from other areas in Vietnam, and 27.6\% from Africa. Most patients (98.5\%) recovered completely. Having severe malaria was a predictor of ETF (AOR 6.96, Cl 2.55-19.02, P < 0.001). No predictor of LTF was identified.

\section{Conclusion}

P. falciparum remains the prevalent malaria parasite. Despite low mortality rate, severe malaria is not rare and is a significant predictor of ETF. To reduce the risk for ETF, studies are needed to examine the effectiveness of combination therapy including parenteral artesunate and a parenteral partner drug for severe malaria. The study alerts the possibility of drug-resistant malaria in Africa and other areas in Vietnam which are known as nonendemic areas of antimalarial drug resistance. A more comprehensive study using molecular technique in these regions is required to completely understand the magnitude of drug-resistant malaria and to design appropriate control strategies.

\section{Introduction}

Malaria is a mosquito-transmitted infection that affects 219 million people and causes 435 thousand deaths worldwide (1). Among the 5 Plasmodium species causing malaria in humans, Plasmodium falciparum is responsible for the most severe forms of malaria (2). In Vietnam, $P$. falciparum is the most prevalent malaria parasite followed by P.vivax (3). Although the prevalence of malaria infection decreased in the last two decades, antimalarial drug resistance has been emerging in Vietnam $(4,5)$. Dihydroartemisinin (DHA) which is an artemisinin derivative has been used for treating malaria infection in Vietnam for more than 20 years (6). In line with the World Health Organization (WHO) recommendation 
(7), artemisinin-based combination therapy (ACT) including DHA and piperaquine (PPQ) has been a mandatory first choice treatment of uncomplicated P. falciparum infection since 2007 (8). However, molecular markers for artemisinin resistance were detected in Cambodia in 2009 and subsequently spread rapidly to other countries in the Greater Mekong Subregion (GMS) including Vietnam $(9,10)$. Moreover, resistance to PPQ has also been confirmed (11). Recently artesunate-pyronaridine has been recommended for uncomplicated $P$. falciparum infection in Dac Nong and Binh Phuoc which are the two provinces mostly affected by DHA-PPQ resistance (12). Diagnostic tests used to identify antimalarial drug resistance such as molecular assays are not always available, especially in low-resource countries (13). Therefore, monitoring response to treatment, mainly parasite clearance time and fever clearance time are recommended in the clinical management of patients with malaria and the surveillance of antimalarial drug resistance (13). The resistance to DHA-PPQ combination therapy causes delayed parasite clearance time (14). The susceptibility of $P$. falciparum to DHA-PPQ combination therapy in southern Vietnam has declined rapidly with the increase in the proportion of patients with parasite clearance time of more than 72 hours (from $38 \%$ in 2012 to $57 \%$ in 2015) (5). To strengthen the current strategies to control the spread and impact of drugresistant $P$. falciparum in Vietnam and other countries in the GMS, it is important to know the current burden of $P$. falciparum infection and the effectiveness of antimalarial drugs in Vietnam. The Hospital for Tropical Diseases (HTD) in Ho Chi Minh City is a tertiary hospital which receives patients with malaria from Central Highland and southern Vietnam including the Vietnam-Cambodia border area. We conducted our study at this hospital to examine the magnitude of $P$. falciparum infection, severe malaria and the response to antimalarial treatment including the patterns of and predictors for treatment failure.

\section{Methods}

\section{Design of the study}

Medical records of all patients with malaria admitted to the HTD between January 2015 and December 2018 were retrieved for review. Information derived from medical records of patients with $P$. falciparum infection was extracted and included demographics (age, sex, job, and residential address), risk factors (blood transfusion, injecting drug use (IDU), travelling to malaria endemic areas domestically and internationally within 14 days before the onset of illness (15), current health conditions (pregnancy, end stage renal disease, cirrhosis, and HIV infection), malaria disease and treatment at previous hospitals and HTD. It is noted that our hospital is the tertiary teaching hospital in southern Vietnam for infectious diseases including malaria. Therefore, as required by the HTD policy, this information must be obtained correctly and entered into the medical record. The data collection was performed by two authors (OKNP and PTN) who are infectious disease specialists and provide malaria treatment to patients at the HTD. Any discrepancies derived from the data collection process were cross-checked by these authors and the other local research assistants who are also clinical doctors until consensus was obtained. It is also noted that if patients have any risk factor for HIV infection, they will be consulted to undertake HIV testing in accordance with the HTD guideline. Information on malaria disease and treatment included admission 
time, number of days of illness at the time of admission, symptoms and signs (fever, anemia, splenomegaly, and hepatomegaly), laboratory tests (malaria microscopy, parasite counts, and aminotransaminases (AST and ALT)), antimalarial and other supportive treatments, response to treatment (number of inpatient days, fever clearance time, parasite clearance time, and early (ETF) and late treatment failure (LTF)) (16), outcomes (recovery and death), and having malaria previously. All laboratory tests including microscopy were performed th the HTD and in line with the national laboratory performance standards. According to the HTD policy, all cases were diagnosed using microscopy. Once the diagnosis is confirmed, patients must be screened for all clinical and laboratory signs of severe malaria. Blood smear for the presence of parasites and parasite density was performed at an interval of six (severe malaria) or 12 hours (uncomplicated malaria) until blood smear showed a negative result for at least two times.

Based on the course of the disease, $P$. falciparum infection was classified into different types of severe malaria in accordance with the WHO guideline for the treatment of malaria and included shock, acute kidney failure, impaired consciousness, jaundice, anemia, hemoglobiuria, acidosis, hyperparasitemia, prostration, convulsion, hypoglycemia and bleeding (7). Regarding shock, the WHO guideline was updated during our study period. In line with the local requirement, shock definition was updated accordingly based on the updated WHO guideline. In detail, for those who admitted to our hospital before September 2016, shock was defined as systolic blood pressure $<50 \mathrm{mmHg}$ in children or $<80 \mathrm{mmHg}$ in adults, with evidence of impaired perfusion (cool peripheries or prolonged capillary refill (17). Since September 2016, shock has been defined as systolic blood pressure $<90 \mathrm{mmHg}$ in adults or a drop of $20 \mathrm{mmHg}$ in systolic pressure by age in children, with evidence of impaired perfusion (18). ETF and LTF were defined according to the WHO recommendation (16) and the distribution of these treatment failures was also examined. However, it is documented that in an area of artemisinin resistance, patients with prolonged parasitemia including those having parasitemia on day 3 with or without axillary temperature $\geq 37.5^{\circ} \mathrm{C}$ respond well to ACT treatment $(14,19)$. Therefore, in our study, ETF did not include these people provided that the study was conducted in an area of artemisinin resistance. ETF included (i) danger signs or severe malaria on day 1,2 or 3, in the presence of parasitemia; or (ii) parasitemia on day 2 higher than on day 0 , irrespective of axillary temperature; and (iii) parasitemia on day $3 \geq 25 \%$ of count on day 0 . ETF and LTF were not routinely assessed for all patients admitted to the HTD. We examined the presence of ETF among our study participants based on the clinical symptoms and laboratory tests. Regarding LTF, although we did not follow up patients on day 28 and day 42 after antimalarial treatment due to the nature of a retrospective study, information on previous malaria infection within 28 days was documented in the medical record as required by the HTD policy. Therefore, patients with LTF in our study were those who met the WHO definition of LTF within 28 days before admission. In detail, LTF includes (i) danger signs or severe malaria in the presence of parasitemia on any day between day 4 and day 28 in patients who did not previously meet any of the criteria of early treatment failure and (ii) presence of parasitemia on any day between day 4 and day 28 (day 42) with axillary temperature $\geq 37.5^{\circ} \mathrm{C}$ in patients who did not previously meet any of the criteria of early treatment failure, or (iii) presence of parasitemia on any day between day 7 and day 28 with axillary temperature $<37.5^{\circ} \mathrm{C}$ in patients who did 
not previously meet any of the criteria of early treatment failure or late clinical failure. Before September 2016, uncomplicated P. falciparum infection was treated with a three-day DHA-PPQ age-based dosing therapy. Severe malaria was treated with intravenous artesunate $2.4 \mathrm{mg} / \mathrm{kg}$ on admission, at 12 hours, and then every 24 hours until oral therapy can be given (17). Since September 2016, although the antimalarial agents remained the same, weight-based dosing has been used for uncomplicated malaria. Regarding severe malaria, the dosage of intravenous artesunate remained unchanged but the maximum duration of artesunate treatment has been set at seven days (18). Given these changes in malaria treatment $(18,20)$, the change in the magnitude of malaria and severe malaria before and after 2017 was also examined. According to the local guideline, in order to be discharged from the hospital, patients must meet the following criteria including receiving a full course of antimalarial therapy, no fever, no severe malaria symptoms, and blood smear negative for parasites for at least two times. Therefore, patients who did not meet all these criteria were followed up in our hospital until discharge. The study protocol was approved by the HTD's Ethics Committee (approval number 65/QD-BVBND) and the Human Research Ethics Committee at UNSW Australia (approval number HC180340).

\section{Statistical analysis}

Data were stored and analyzed using SPSS version 22 (IBM). For comparison, a calculation of 95\% confidence interval $(\mathrm{Cl})$ for the prevalence rate of $P$. falciparum infection was performed based on the number of subjects with malaria infection and the point estimate of the prevalence of $P$. falciparum infection. Similar calculations were performed for the prevalence rates of severe malaria, ETF and LTF. Chi-square, chi-square for trend and Fisher's Exact tests were used to compare categorical data. Student's t-test was used to compare continuous data. Multinomial logistic regression models were developed to test predictors of ETF and LTF. Independent variables including age, severe malaria, and acquiring malaria before 2017 were entered into the ETF model. Similarly, age, severe malaria, and patients with parasitemia 72 hours of treatment were entered into the LTF model. The significant level was set at $P \leq$ 0.05 .

\section{Results}

\section{Demographic characteristics of study participants}

There were 443 malaria infected patients admitted to our hospital between January 2015 and December 2018. Of these patients $P$. falciparum malaria accounted for $59.8 \%$ (265/443, $\mathrm{Cl} 55.1 \%-64.4 \%)$. Among 265 P. falciparum infected patients, nearly half $(44.5 \%, 118 / 265)$ of them acquired infection before 2017 and most of them (83.4\%) were male with the mean age of $35.3 \pm 13.1$ years (Table 1). More than half $(56.3 \%)$ of patients had jobs related to the forest or worked in malaria endemic areas, including mountain farmers $(29.1 \%)$, forest workers $(8.7 \%)$, forest ranger $(1.9 \%)$, healthcare worker working in the forest (0.4\%) and working in Cambodia and Africa (16.2\%). Having previous blood transfusion was documented in one patient and each of IDU and pregnancy was noted in the other two patients. No one had HIV infection. Nearly half $(44.9 \%, 119 / 265)$ of study participants lived in malaria endemic areas and $36.6 \%$ 
$(97 / 265)$ travelled to endemic areas within 14 days prior to the onset of disease. The number of cases was peaked during the period between November and March annually (Fig. 1). 
Table 1

Demographic characteristics of $265 P$. falciparum infected patients receiving treatment at the Hospital for Tropical Diseases between 2015 and 2018

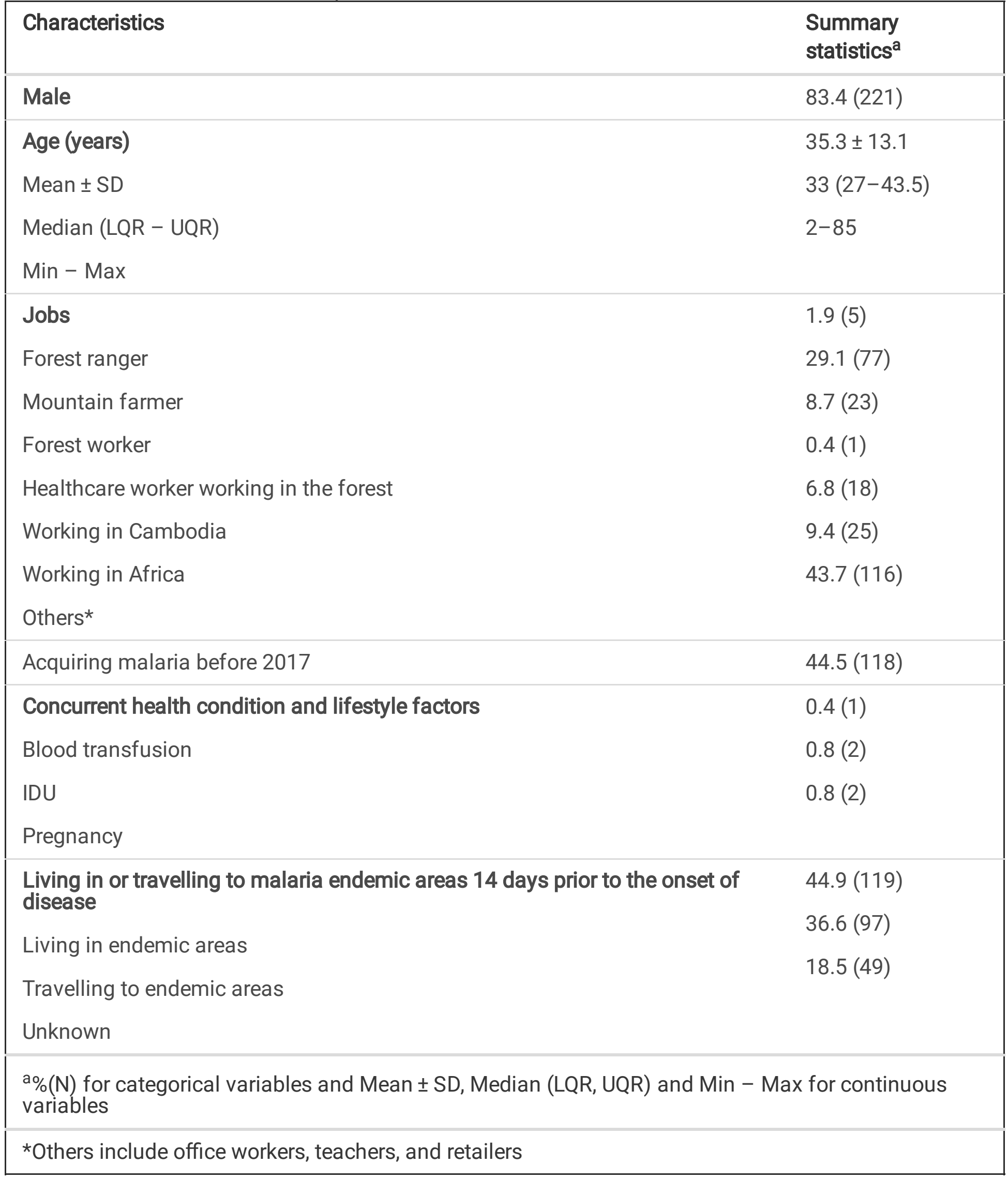




\section{Clinical manifestations of P. falciparum infected patients}

Almost all patients $(99.6 \%, 264 / 265)$ had fever on admission, while only $0.8 \%$ had anemia, $3 \%$ had splenomegaly, and $10.2 \%$ had hepatomegaly (Table 2$)$. There were $84(31.7 \%)$ patients receiving treatment at previous hospitals. Among these patients, three received oral quinine at previous hospitals, of whom two used quinine for two days and one used seven days. Parasitemia still persisted in all of them at the time of admission. At our hospital, two cases taking two-day quinine therapy were treated with a three-day DHA-PPQ therapy and a case taking seven-day quinine therapy was treated with a fourday intravenous artesunate followed by three-day DHA-PPQ therapy, of whom the latter developed ETF. All of them completely recovered in the end. None of our participants was treated with quinine at our hospital. Fifty-eight patients $(21.9 \%, 58 / 265, \mathrm{Cl} 17.1 \%-27.4 \%)$ had severe malaria, of whom single manifestation accounted for $77.6 \%$ (45/58, $\mathrm{Cl} 64.7 \% 87.5 \%)$. The most common manifestations included jaundice $(24.1 \%, 14 / 58)$, impaired consciousness $(20.7 \%, 12 / 58)$, and shock $(8.6 \%, 5 / 58)$. All five patients developing shock were adults, of whom three admitted to our hospital before September 2016 and all had a systolic blood pressure of $\leq 80 \mathrm{mmHg}$ at the time of shock. A quarter $(25.3 \%, 67 / 265)$ of patients had developed illness for more than seven days at the time of admission. One third of study participants had AST $(31.3 \%, 83 / 265)$ and ALT $(30.2 \%, 80 / 265)>40 \mathrm{U} / \mathrm{L}$, of whom the mean AST and ALT levels were $99.6 \pm 98.8 \mathrm{U} / \mathrm{L}$ and $98.1 \pm 60.2 \mathrm{U} / \mathrm{L}$, respectively. 
Table 2

Clinical patterns of P.falciparum infection among 265 infected patients receiving treatment at the Hospital for Tropical Diseases between 2015 and 2018

\section{Characteristics}

Signs and symptoms

Fever on admission

Anemia

Splenomegaly

Hepatomegaly

Severe malaria

Manifestations of severe malaria $(n=58)$

Single manifestations

Shock

Acute kidney failure

Impaired consciousness*

Jaundice

Anemia

Hemoglobinuria

Acidosis

Hyperparasitemia

Prostration

Convulsions

Hypoglycemia

Bleeding
Summary statistics ${ }^{a}$

99.6 (264)

$0.8(2)$

3 (8)

$10.2(27)$
$21.9(58)$

$77.6(45)$

$8.6(5)$

$6.9(4)$

$20.7(12)$

24.1 (14)

$6.9(4)$

$1.7(1)$

$1.7(1)$

$6.9(4)$

0

0

0

0

$22.4(13)$

Mixed manifestations (i.e. patients had more than one severe manifestation)

Receiving treatment at previous hospital

$31.7(84)$

a\%(N) for categorical variables and Mean \pm SD, Median (LQR, UQR) and Min - Max for continuous variables

* Glasgow coma score $<11$ in adults. There were 19 children patients in this study and none of them had impaired consciousness 


\section{Characteristics}

Summary statistics ${ }^{a}$

Diagnosis at previous hospital $(n=84)$

$78.6(66)$

Malaria

$21.4(18)$

Others (i.e. dengue infection and septicemia)

Number of days of illness $>7$ days at the time of admission (days)

$25.3(67)$

Number of days of illness at the time of admission (days)

$6.6 \pm 5.1$

Mean \pm SD

$5(3,8)$

Median (LQR, UQR)

$1-30$

Min - Max

AST $>40 \mathrm{U} / \mathrm{L}$

Highest AST recorded $(\mathrm{U} / \mathrm{L})(\mathrm{n}=83)$

$99.6 \pm 98.8$

Mean \pm SD

$67(53,116)$

Median (LQR, UQR)

41-864

Min - Max

$\mathrm{ALT}>40 \mathrm{U} / \mathrm{L}$

$30.2(80)$

Highest ALT recorded $(U / L)(n=80)$

$98.1 \pm 60.2$

Mean \pm SD

$76.5(52.5,121.5)$

Median (LQR, UQR)

43-308

Min - Max

a\%(N) for categorical variables and Mean \pm SD, Median (LQR, UQR) and Min - Max for continuous variables

* Glasgow coma score $<11$ in adults. There were 19 children patients in this study and none of them had impaired consciousness

\section{Treatment, response to treatment, and outcomes of $P$. falciparum infected patients}

In addition to antimalarial treatment, 5.7\% (15/265) of patients received red blood cell transfusion, 4.9\% $(13 / 265)$ received mechanical ventilation, and $3 \%(8 / 265)$ undertook hemodialysis (Table 3$)$. Hospital acquired infection was developed in 11 (4.2\%) patients, of whom six developed pneumonia, four developed urinary tract infection, and one developed septicemia. Comorbidities were found in three patients including one with hypertension and type 2 diabetes, one with Chronic obstructive pulmonary disease (COPD), and one with immune thrombocytopenia. Ten patients developed severe malaria 
including one with shock, two with acute kidney failure, three with impaired consciousness, two with jaundice, and two with mixed manifestations. One patient died. Extended-Spectrum Beta-Lactamase (ESBL)-producing E. coli (two patients), Klebsiella pneumoniae (one patient), and Pseudomonas aeruginosa (two patients) were detected. The infectious agent was not detected in the remaining six patients. 
Table 3

Treatment and outcome of 265 P. falciparum infected patients receiving treatment at the Hospital for Tropical Diseases between 2015 and 2018

\section{Characteristics}

Summary statistics ${ }^{a}$

Mechanical ventilation

$4.9(13)$

Hemodialysis

$3(8)$

Red blood cell transfusion

$5.7(15)$

Hospital acquired infection (HAl)

$4.2(11)$

Patients with fever 3 days of treatment

$13.2(35)$

Fever clearance times (days)

$2.4 \pm 1.5$

Mean \pm SD

$2(1,3)$

Median (LQR, UQR)

$1-14$

Min - Max

Patients with parasitemia 72 hours of treatment

$15.1(40)$

Parasite clearance times (hours)

$53 \pm 30.8$

Mean \pm SD

$48(30,72)$

Median (LQR, UQR)

$1-228$

Min - Max

Early treatment failure

$7.2(19)$

Distribution of early treatment failure

$4.7(7)$

Vietnam

$12.9(8)$

Binh Phuoc and Central Highland $(n=148)$

$7.7(2)$

Other areas $(n=62)$

$6.9(2)$

Cambodia $(n=26)$

Africa $(n=29)$

Patients with parasitaemia on day 3 with axillary temperature $\geq 37.5^{\circ} \mathrm{C}$

$3(8)$

Late treatment failure

$19.2(51)$

a\%(N) for categorical variables and Mean \pm SD, Median (LQR, UQR) and Min - Max for continuous variables 


\section{Characteristics}

Distribution of late treatment failure

Vietnam

Binh Phuoc and Central Highland $(n=148)$

Other areas $(n=62)$

Cambodia $(n=26)$

Africa $(n=29)$

Number of inpatient days

Mean \pm SD

Median (LQR, UQR)

Min - Max

ICU admission

Response to treatment

Recovery

Death
$16.2(24)$

$22.6(14)$

$19.2(5)$

$27.6(8)$
$5.7 \pm 3.5$

$5(4,6)$

$1-28$

$10.9(29)$

$98.5(261)$

$1.5(4)$

$\mathrm{a} \%(\mathrm{~N})$ for categorical variables and Mean $\pm \mathrm{SD}$, Median (LQR, UQR) and Min - Max for continuous variables

Thirty-five (13.2\%) and 40 (15.1\%) patients had fever and parasitemia after 3 days of antimalarial treatment, respectively. The mean fever clearance time was $2.4 \pm 1.5$ days, and the mean parasite clearance time was $53 \pm 30.8$ hours. ETF and LTF were documented in $19(7.2 \%, \mathrm{Cl} 4.6 \%-10.9 \%)$ and 51 $(19.2 \%, \mathrm{Cl} 14.7 \%-24.5 \%)$ patients, respectively. ETF accounted for $4.7 \%(\mathrm{Cl} 2.3 \%-9.4 \%)$ of patients coming from or travelling to Binh Phuoc and Central Highland, $12.9 \%(\mathrm{Cl} 6.7 \%-23.5 \%)$ from other areas in Vietnam, 7.7\% (Cl 2.1\% - 24.1\%) from Cambodia and 6.9\% (Cl 1.9\% - 22.0\%) from Africa. Similarly, the proportion of LTF was $16.2 \%$ (Cl 11.2\% - 23.0\%) among patients coming from or travelling to Binh Phuoc and Central Highland, 22.5\% (Cl 14.0\% - 34.4\%) from other areas in Vietnam, 19.3\% (Cl 8.5\% - 37.9\%) from Cambodia and $27.6 \%(\mathrm{Cl} 14.7 \%$ - 45.7\%) from Africa. The proportion of patients with parasitemia on day 3 with axillary temperature $\geq 37.5^{\circ} \mathrm{C}$ was $3 \%(8 / 265)$. The mean number of inpatient days was $5.7 \pm 3.5$. There were $29(10.9 \%)$ patients required ICU admission and the mean ICU length of stay was $2.9 \pm 1.5$ days. Most patients $(98.5 \%, 261 / 265)$ recovered completely and $6.9 \%(4 / 58)$ of patients with severe malaria died. There was no association between having parasitemia 72 hours of treatment and response to treatment $(P=0.48)$. Among 207 patients with uncomplicated malaria, no one died or needed mechanical ventilation and hemodialysis, and one (1\%) developed hospital acquired infection. Among 58 
patients with severe malaria, eight required hemodialysis, 13 required mechanical ventilation, and nine developed hospital acquired infection.

Among 29 patients coming from or travelling to Africa, $96.6 \%$ (28/29) was male with a median age of $35.1 \pm 10.2$ years (Table 4 ). The mean number of days of illness at the time of admission and the mean number of inpatient days were $6.1 \pm 5.5$ and $5.1 \pm 3.8$ days, respectively. Three (10.3\%) and two (6.9\%) patients had fever and parasitemia 3 days of antimalarial treatment, respectively. Severe malaria was documented in $8(27.6 \%)$ patients, of whom one developed shock, one developed impaired consciousness, one developed hyperparasitemia, two developed jaundice, and three developed mixed manifestations. ETF and LTF were recorded in $2(6.9 \%)$ and 8 (27.6\%) patients, respectively. Three (10.3\%) patients received red blood cell transfusion, two (6.9\%) undertook hemodialysis, and three received mechanical ventilation. Two patients died, of whom, one patient was 48 years old with atrial fibrillation and type 2 diabetes who admitted to our hospital on day seven of illness with shock and severe metabolic acidosis and another patient was 28 years old who admitted to our hospital on day four of illness with severe metabolic acidosis and impaired consciousness. 
Table 4

Demographic characteristics, treatment, and outcome of 29 P. falciparum infected patients from Africa

\section{Characteristics}

Male

Age

Mean \pm SD

Median (LQR, UQR)

Min - Max

Number of days of illness at the time of admission (days)

Mean \pm SD

Median (LQR, UQR)

Min - Max

Number of inpatient days

Mean \pm SD

Median (LQR, UQR)

Min - Max

Patients with parasitemia 72 hours of treatment

Patients with fever 3 days of treatment

Severe malaria

Manifestation of severe malaria $(n=8)$

Shock

Impaired consciousness

Jaundice

Hyperparasitemia

Mixed manifestations (i.e. patients had more than one severe manifestation)

Early treatment failure

Late treatment failure

Red blood cell transfusion
Summary statistics ${ }^{a}$

$96.6(28)$

$35.1 \pm 10.2$

$32(28,43)$

$22-69$

$5(3.5,7)$

$1-30$

$5.1 \pm 3.8$

$4(3,6)$

2-23

6.9 (2)

10.3 (3)

27.6 (8)

3.4 (1)

3.4 (1)

6.9 (2)

3.4 (1)

$10.2(3)$

6.9 (2)

27.6 (8)

$10.3(3)$

a\%(N) for categorical variables and Mean \pm SD, Median (LQR, UQR) and Min - Max for continuous variables 


\begin{tabular}{|lc|}
\hline Characteristics & Summary statistics $^{\mathbf{a}}$ \\
\hline Hemodialysis & $6.9(2)$ \\
\hline Mechanical ventilation & $10.3(3)$ \\
\hline Death & $6.9(2)$ \\
\hline $\begin{array}{l}\text { a\%(N) for categorical variables and Mean } \pm \text { SD, Median (LQR, UQR) and Min - Max for continuous } \\
\text { variables }\end{array}$ \\
\hline
\end{tabular}

\section{Predictors for severe malaria}

Severe malaria was statistically associated with receiving treatment at previous hospitals $(P<0.001)$, hepatomegaly $(P<0.001)$ and number of inpatient days $(P<0.001)($ Table 5$)$. There were no association between severe malaria and gender, age, BMI, number of days of illness, acquiring malaria before 2017, pregnancy, fever on admission, splenomegaly, and anemia $(P>0.05)$. 
Table 5

Association between patterns of malaria infection and demographic and clinical characteristics

\begin{tabular}{|c|c|c|c|c|}
\hline Characteristics $^{a}$ & $\begin{array}{l}\text { Severe } \\
\text { malaria } \\
(n=58)\end{array}$ & $\begin{array}{l}\text { Uncomplicated } \\
\text { malaria } \\
(n=207)\end{array}$ & $\begin{array}{l}\mathrm{P} \\
\text { value* }\end{array}$ & OR (Cl) \\
\hline Age (years) & $37.3 \pm 14.2$ & $34.7 \pm 12.7$ & 0.17 & \\
\hline Male & $82.8(48)$ & $83.6(173)$ & 0.9 & \\
\hline BMI & $21.5 \pm 3.3$ & $21.4 \pm 3$ & 0.87 & \\
\hline Number of days of illness & $6.8 \pm 4.4$ & $6.3 \pm 5.2$ & & \\
\hline $\begin{array}{l}\text { Receiving treatment in previous } \\
\text { hospital }\end{array}$ & $16(27.6 \%)$ & $26(12.6 \%)$ & $<001$ & $\begin{array}{l}2.7(1.3- \\
5.3)\end{array}$ \\
\hline Acquiring malaria before 2017 & $46.6(27)$ & $44(91)$ & 0.72 & \\
\hline Pregnancy & $1.7(1)$ & $0.5(1)$ & 0.3 & \\
\hline Fever on admission & $100(58)$ & $99.5(206)$ & 0.6 & \\
\hline Hepatomegaly & $31(18)$ & $4.3(9)$ & $<001$ & $\begin{array}{l}9.9(4.2- \\
23.6)\end{array}$ \\
\hline Splenomegaly & $5.2(3)$ & $2.4(5)$ & 0.3 & \\
\hline Anemia & $1.7(1)$ & $0.5(1)$ & 0.3 & \\
\hline Number of inpatient days & $7.8 \pm 4.2$ & $5 \pm 3$ & $<0.001$ & \\
\hline \multicolumn{5}{|c|}{ a\%(N) for categorical variables and Mean \pm SD for continuous variables } \\
\hline
\end{tabular}

\section{Predictors for early and late treatment failures}

Patients with severe malaria were significantly more likely to develop ETF $(P<0.001,0 R 7.5, \mathrm{Cl} 2.8-20.0)$ and less likely to develop LTF $(P=0.044, O R 0.44, C l 0.19-0.99)$ compared with those who did not have severe malaria (Table 6). Gender, living in or travelling to malaria endemic areas 14 days prior to the onset of disease, number of days of illness $>7$ days at the time of admission, acquiring malaria before 2017 and hyperparasitemia $(P>0.05)$ were not predictors of ETF and LTF. Similarly, having parasitemia 72 hours of treatment and fever 3 days of treatment $(P>0.05)$ were not predictors of LTF. There is an association between age and ETF $(P=0.027)$, while there is no association between age and LTF $(P=$ 0.054). 
Table 6

Unadjusted predictors tested for early and late treatment failures

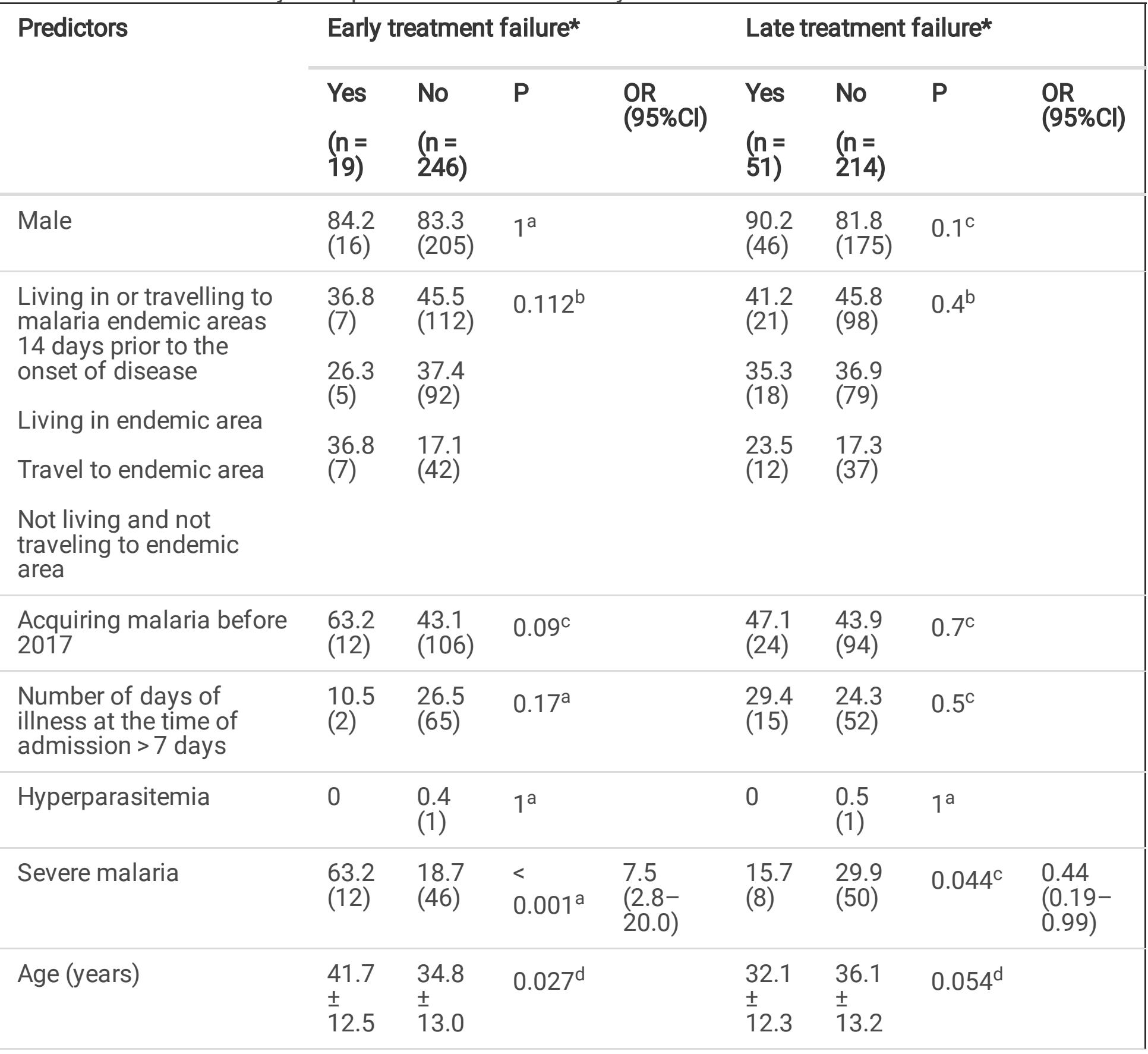

${ }^{*} \%(\mathrm{~N})$ for categorical variables and Mean \pm SD for continuous variables

${ }^{a}$ Fisher's Exact test

${ }^{b}$ Chi-square for trend test

${ }^{\mathrm{c}}$ Chi-square test

d Student's t test 
Predictors

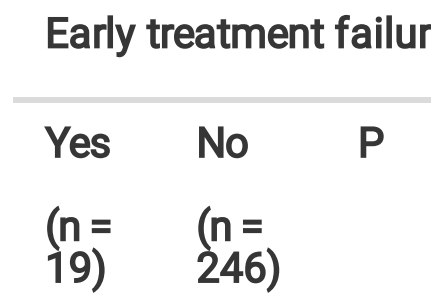

\section{Patients with}

parasitemia 72 hours of

treatment

Patients with fever 3

days of treatment
Late treatment failure*

$\begin{array}{lllll}\text { OR } & \text { Yes } & \text { No } & P & \text { OR } \\ (95 \% \mathrm{Cl}) & & & & (95 \% \mathrm{Cl}) \\ & (\mathrm{n}= & (\mathrm{n}= & & \\ & 51) & 214) & & \end{array}$

$\begin{array}{llll}25 & 13.5 & 0.047^{c} & 2.14 \\ (12) & (28) & & (0.99- \\ & & & 4.61)\end{array}$

$14.6 \quad 15.8$

(6) (29)

$0.9^{c}$

* $\%(\mathrm{~N})$ for categorical variables and Mean \pm SD for continuous variables

a Fisher's Exact test

${ }^{b}$ Chi-square for trend test

${ }^{\mathrm{c}}$ Chi-square test

${ }^{d}$ Student's t test

\section{Model for the prediction of early and late treatment failures}

No predictor for ETF was identified other than having severe malaria (Adjusted odds ratio (AOR) 6.96, Cl 2.55-19.02, $\mathrm{P}<0.001$ ) (Table 7). No predictor for LTF was identified.

Table 7

Multinominal logistic regression analysis for predictors of early and late treatment failures

\begin{tabular}{|lllll|}
\hline Predictors & \multicolumn{2}{c|}{ Early treatment failure } & \multicolumn{2}{c|}{ Late treatment failure } \\
\cline { 2 - 6 } & $\begin{array}{l}\mathbf{P} \\
\text { value }\end{array}$ & $\begin{array}{l}\text { Adjusted OR } \\
(95 \% \mathrm{Cl})\end{array}$ & $\begin{array}{l}\mathbf{P} \\
\text { value }\end{array}$ & $\begin{array}{l}\text { Adjusted OR } \\
(95 \% \mathrm{Cl})\end{array}$ \\
\hline Age (years) & 0.062 & $\begin{array}{l}1.04(0.998- \\
1.073)\end{array}$ & 0.11 & $0.98(0.95-1.01)$ \\
\hline Severe malaria & $<$ & $\begin{array}{l}6.96(2.55- \\
19.02)\end{array}$ & 0.094 & $0.49(0.21-1.13)$ \\
\hline Acquiring malaria before 2017 & 0.001 & & & \\
\hline $\begin{array}{l}\text { Patients with parasitemia 72 hours of } \\
\text { treatment }\end{array}$ & 0.096 & $2.38(0.86-6.59)$ & & \\
\hline
\end{tabular}

\section{Discussion}

A total of 433 patients with malaria admitted to the HTD between 2015 and 2018. Of these 433 patients more than half $(59.8 \%, \mathrm{Cl} 55.1 \%-64.4 \%)$ acquired $P$. falciparum infection. Our finding is lower than the 
rate of $98 \%(\mathrm{Cl} 97.5 \%-98.5 \%)$ reported from a provincial hospital in southern Vietnam in $1990(21)$. It is impossible to completely understand the reason of this change due to the difference in the study clinics and referral patterns. However, Goldlust et al. have found that the adoption of ACTs may have influenced the decline of malaria including $P$. falciparum in Vietnam (22). We found that most patients were male in labor age and more than half $(56.3 \%)$ of them had jobs related to the forest or worked in areas which are known as malaria endemic areas in Vietnam, Cambodia and Africa $(23,24)$. Additionally, more than $80 \%$ of patients lived in or traveled to malaria endemic areas within 14 days before the onset of disease which is an established risk for malaria infection (15). These findings were not surprised since according to Vietnamese culture, breadwinner is perceived as the man's role in the family, and therefore more men tend to be exposed to malaria infection than women when they work in or travel to malaria endemic areas (25). At this stage, most malaria morbidities and mortalities occur in 21 out of 58 provinces, of which forested areas of provinces located in Central and Central-Southern Vietnam account for the highest malaria burden in Vietnam (26). In our study, P. falciparum infection was recorded throughout the year and peaked during the months from November to March of the following year. In Vietnam, Lunar New Year holiday which is the most important holiday usually occurs in the second half of this period. Based on our experience, to financially prepare for the holiday many people travel to malaria endemic areas to work in this period in response to the increase in the number of seasonal jobs in these areas.

Blood transfusion and IDU are considered as malaria risk factors $(27,28)$. We found only one patient who was suspected to have malaria infection from blood transfusion. Two other cases were injecting drug users who may have shared needles and syringes in the days before their illness developed. Pregnancy is a high risk for developing severe malaria $(29,30)$. Fortunately, two pregnant patients in our study had uncomplicated malaria.

According to the WHO definition of severe falciparum malaria (31) and the Vietnam Ministry of Health guidelines for the management of malaria infection (18), patients were classified into uncomplicated and severe malaria groups. In our study, severe malaria accounted for $21.9 \%(\mathrm{Cl} 17.1 \%-27.4 \%)(21,32,33)$. We found an association between severe malaria and prolonged inpatient days. This is probably due to the prolonged antimalarial therapy and different severe manifestations which required additional supportive treatment as compared with uncomplicated malaria (34). Similar to another report (35), we observed an association between hepatomegaly and severe malaria in our study. We also found that severe malaria was associated with receiving treatment at previous hospitals among our study participants. In detail, $62.5 \%$ (10/16) of study participants receiving treatment at previous hospitals were incorrectly diagnosed as having dengue hemorrhagic fever or septicemia. It is well documented that misdiagnosis of malaria at previous hospitals leads to delayed antimalarial treatment, and thus may facilitate severe malaria (36-38). In light of this, clinicians should pay more attention on the patient's epidemiological evidence of malaria to prevent misdiagnosis of this disease.

Regarding the clinical symptoms, similar to previous reports $(33,39)$, fever was frequent $(99.6 \%)$, while anemia was rare $(0.8 \%)$ in our study. However, splenomegaly was reported to be a common symptom among $P$. falciparum infected patients, while hepatomegaly was not mentioned in another study (39). We

Page 20/30 
detected splenomegaly in only $3 \%$ and hepatomegaly in up to $10.2 \%$ of study participants. Among 58 cases with severe malaria, the most common manifestations were impaired consciousness $(20.7 \%)$, jaundice $(24.1 \%)$ and mixed manifestations (22.4\%). Other manifestations of severe malaria included anemia, renal failure, hyperparasitemia and shock which are similar to other reports $(21,33)$. Although mild elevations of transaminases are common in Plasmodium infection, particularly in $P$. falciparum infection, this condition is usually transient $(40,41)$. About $30 \%$ of our patients had transaminase higher than $40 \mathrm{U} / \mathrm{L}$. Treatment delay of seven days or more is documented to be a risk for severe malaria with multi-organ failure (42). We noted that $25 \%$ of study population admitted to our hospital after seven days of illness and this included some patients receiving treatment from previous hospitals. In our study, more than one fifth of study participants receiving treatment from previous hospitals had been misdiagnosed as having dengue infection or septicemia. An inappropriate therapy provided in previous hospitals could aggravate patient's condition (43). This could explain why some patients who were admitted to the HTD after 7 days of disease developed severe malaria. Therefore, clinicians must be more alerted to malaria infection when patients have a travel history to endemic areas (43). In addition, severe malaria should be suspected if patients were under inappropriate treatment at previous hospitals (44).

Having a prolonged fever after receiving antimalarial treatment is considered as a predictor of treatment failure $(45,46)$. In addition, prolonged fever indirectly reflects the activity of parasite in the human body and consequently indicates antimalarial drug resistance (45). In our study, $13.2 \%$ of patients had fever longer than 3 days after using DHA-PPQ which is one of the most effective ACTs (7). This suggests a reduced susceptibility of DHA-PPQ in our study population. Parasite clearance time is also useful to detect artemisinin resistance $(45,47)$. Binh Phuoc province is an endemic area of artemisinin resistance in Vietnam (48). A study showed that the prevalence of positive blood smear for asexual parasitemia 72 hours after treatment initiation in Binh Phuoc was up to $75 \%$ (49). In our study, nearly $50 \%$ of patients coming from Binh Phuoc and $15.1 \%$ of patients having parasitemia 72 hours of treatment. However, we were unable to perform molecular tests to detect K13 mutations among these patients to confirm artemisinin resistance due to the nature of a retrospective study.

We found that the rates of ETF and LTF in other areas in Vietnam were not different to those in Binh Phuoc-Central Highland where DHA and PPQ resistance has been confirmed, this raises a big concern about the spread of malaria parasites that are resistant to both DHA and PPQ to other areas in Vietnam. It has been documented that artemisinin resistance has emerged in the GMS, followed by ACTs' failure, because both artemisinin and partner drug have decreased their susceptibility (48). According to a recent study examining the efficacy of DHA-PPQ combination in this region, the prevalence of plasmepsin $2 / 3$ amplification which is a marker of PPQ resistance in Binh Phuoc-Vietnam was $77 \%$ and the efficacy of DHA-PPQ on day 42 was only $47.1 \%$, while there was only $7 \%$ of cases having fever on the day of recrudescent infection (49). Given the high prevalence of marker of PPQ resistance, the low efficacy of DHA-PPQ treatment and unclear clinical sign of recrudescent infection, clinically detection of this antimalarial drug resistance is challenging. In light of this, antimalarial drug resistance may have spread beyond Binh Phuoc province in Vietnam. Besides, $P$. falciparum infection is prevalent in Africa (1). The proportions of patients from Africa developing ETF (6.9\%) and LTF (27.6\%) in our study raise a concern 
about the risk of artemisinin resistance in Africa. Indeed, although the local artemisinin resistance has not been confirmed, the unexplained slow parasite clearance times (50) and residual submicroscopic parasitemia after artemisinin-combination therapy (51) have been reported in Uganda and Kenya, respectively.

DHA-PPQ combination has been widely used as a first-choice therapy for $P$. falciparum infection in many countries including Vietnam (52). However, the spread of artemisinin and partner drug resistance has caused high treatment failure rates to this combination (53) and subsequently threatens the success of malaria control and elimination (9). In Vietnam, before 2016, DHA-PPQ treatment dose depended on patient's age (54). However, many studies have showed that PPQ under-dosing ( $<48 \mathrm{mg} / \mathrm{kg})$ is an important factor for recrudescent parasitemia $(52,55)$. It is documented that the underdose of DHA-PPQ increases recrudescent parasitemia and sequentially increases treatment failure (52). Indeed, the underdose of $P P Q$ is a significant predictor for recrudescence with the risk increasing by $13 \%(95 \% \mathrm{Cl}$ $5.0-21 \%$ ) for every $5 \mathrm{mg} / \mathrm{kg}$ decrease in dose (52). A pharmacological modelling study has found that compared with DHA-PPQ weight-based doing, age-based dosing increases the risk of underdose of DHA$P P Q$, and thus risk of malaria treatment failure and recrudescence (56). In addition, several studies have found that the DHA-PPQ weigh-based dosing enhances prolonged useful therapeutic life including maximizing the likelihood of rapid clinical and parasitological cure and minimize transmission and retard drug resistance (56-59). In order to optimize the effectiveness of DHA-PPQ, the WHO recommends treatment with 3 days of DHA-PPQ weight-based dosing to cover at least two asexual life circles of $P$. falciparum (7). In September 2016, the Vietnam Ministry of Health adopted the WHO guideline which has been widely used since 2017 (18). However, we did not find any difference in the proportion of ETF and LFT before and after 2017 in our study. There was an increase in the burden of DHA-PPQ resistant parasites in the GMS including Binh Phuoc- Vietnam between 2011 and 2018 (49). This increased burden of parasites carrying mutations is probably responsible for the unchanged proportions of ETF and LTF before and after 2017 in our study even though the new treatment guideline has been utilized.

A study on African children with uncomplicated falciparum malaria demonstrated the association between age less than 2 years and delayed parasite clearance (60). In addition, hyperparasitemia (> $50.000 / \mathrm{ul}$ ) was also reported as a predictor of delayed parasite clearance (60). We did not find any association between both two treatment failure types and age and hyperparasitemia. However, we found that having severe malaria was a significant predictor of ETF $(P<0.001, A O R 6.96, \mathrm{Cl} 2.55-19.02)$ which has not been reported elsewhere. Similar to other countries, in Vietnam, treatment of severe malaria is at least 24 hours of parenteral artesunate single-therapy plus a three-day oral DHA-PPQ combination therapy when the patient can tolerate oral therapy (18). The presence of artemisinin resistance which can lead to slow parasite clearance in Southeast Asia including Vietnam $(9,61)$ threatens the effectiveness of the initial parenteral artesunate single-therapy for severe malaria. We wonder if the variation in time to receive DHA-PPQ combination therapy and the above-mentioned DHA-PPQ resistance may facilitate ETF among patients with severe malaria. In light of this, to reduce the risk for ETF, more robust studies are needed to examine the effectiveness of an early initiation of combination therapy including parenteral artesunate and a parenteral partner drug for severe malaria. It is important to re-evaluate the 
effectiveness of the current WHO recommended antimalarial therapy for both uncomplicated and severe malaria as well as to develop new intramuscular or parenteral antimalarial drugs in the context of antimalarial drug resistance in the GMS.

Our study had some limitations. First, this is a single center study, and thus we may have missed patients with falciparum infection receiving treatment at other hospitals but were not referred to the HTD. However, the HTD is the only major tertiary teaching hospital for infectious diseases including malaria in southern Vietnam and receives not only local patients, but also patients from the other countries. This would enhance the generalizability of the study findings. Our study interval included the period when the WHO's new treatment guideline was adopted. This allowed us to examine the change in the patterns of treatment failure in response to the utilization of the new guideline. Second, we were unable to perform molecular tests to further examine the magnitude of parasites carrying mutations due to the nature of a retrospective study. Third, we were unable to examine the presence of parasitemia on day 28 for all patients due to the same reason. We may have missed some cases who had parasitemia on day 28 but did not exhibit any clinical symptom. Therefore, the true burden of LTF may be underestimated.

\section{Conclusions}

P. falciparum remains the prevalent malaria parasite in Vietnam. Despite the low mortality rate, severe falciparum malaria is not rare and having severe malaria is a significant predictor of ETF. To reduce the risk for ETF, studies are needed to examine the effectiveness of combination therapy including parenteral artesunate and a parenteral partner drug for severe malaria. The study alerts the risk of the spread of $P$. falciparum that is resistant to both DHA and PPQ to other areas in Vietnam and Africa which are currently known as nonendemic areas of antimalarial drug resistance. A more comprehensive epidemiological survey using molecular technique in these regions is required to completely understand the magnitude of antimalarial drug resistance and to design appropriate control strategies.

\section{Abbreviations}

95\% confidence interval: $\mathrm{Cl}$

Artemisinin-based combination therapy: ACT

Adjusted odds ratio: AOR

Aminotransaminases AST and ALT

Chronic obstructive pulmonary disease: COPD

Dihydroartemisinin: DHA

Early treatment failure: ETF

Page 23/30 
Greater Mekong Subregion: GMS

Hospital for Tropical Diseases: HTD

Injecting drug use: IDU

Piperaquine: PPQ

World Health Organization: WHO

\section{Declarations}

Ethics approval and consent to participate: The study protocol was approved by the HTD's Ethics Committee (approval number 65/QD-BVBND) and the Human Research Ethics Committee at UNSW Australia (approval number HC180340).

Consent for publication: Not applicable

Availability of data and materials: The datasets used during the current study are available from the corresponding author on reasonable request

Acknowledgements: We thank Drs Cao Xuan Nam and Nguyen Thien Duc for cross-checking the validity of data.

Funding: Minh Cuong Duong is funded by the Australian Government through the Australian Alumni Grants Fund

Competing interests: No relevant disclosures

Authors' contributions: conceptualization: MCD, OKNP and PNH; data acquisition: OKNP, MCD, PTN, VVCN and $\mathrm{PNH}$; formal analysis and writing original draft: $\mathrm{MCD}, \mathrm{OKNP}$ and $\mathrm{PNH}$; reviewing and editing: $\mathrm{MCD}$, OKNP, PTN, VVCN and PNH.

\section{References}

1. World Health Organization. Malaria Geneva, Switzerland: WHO; 2019 [cited 201929 July]. Available from: https://www.who.int/news-room/fact-sheets/detail/malaria.

2. Orish V, Afutu L, Ayodele O, Likaj L, Marinkovic A, Sanyaolu A. A 4-Day Incubation Period of Plasmodium falciparum Infection in a Nonimmune Patient in Ghana: A Case Report. Open Forum Infect Dis. 2019;6(1):ofy169-ofy.

3. Hong NV, Delgado-Ratto C, Thanh PV, Van den Eede P, Guetens P, Binh NT, et al. Population Genetics of Plasmodium vivax in Four Rural Communities in Central Vietnam. PLoS neglected tropical diseases. 
2016;10(2):e0004434.

4. Goldlust SM, Thuan PD, Giang DDH, Thang ND, Thwaites GE, Farrar J, et al. The decline of malaria in Vietnam, 1991-2014. Malaria Journal. 2018;17(1):226.

5. Thanh NV, Thuy-Nhien N, Tuyen NTK, Tong NT, Nha-Ca NT, Dong LT, et al. Rapid decline in the susceptibility of Plasmodium falciparum to dihydroartemisinin-piperaquine in the south of Vietnam. Malaria Journal. 2017;16(1):27.

6. Tran TH, Dolecek C, Pham PM, Nguyen TD, Nguyen TT, Le HT, et al. Dihydroartemisinin-piperaquine against multidrug-resistant Plasmodium falciparum malaria in Vietnam: randomised clinical trial. Lancet (London, England). 2004;363(9402):18-22.

7. World Health Organization. Guidelines for the treatment of malaria. Third edition Geneva, Switzerland: WHO; 2015 [cited 20195 August]. Available from:

https://www.who.int/malaria/publications/atoz/9789241549127/en/.

8. Vietnam Minstry of Health. Guideline for diagnosis and treatment of malaria [in Vietnamese]: VietnamMinstryofHealth; 2007 [cited 2019 Oct 14]. Available from: https://vanbanphapluat.co/quyetdinh-339-qd-byt-huong-dan-chuan-doan-dieu-tri-benh-sot-ret.

9. Imwong M, Suwannasin K, Kunasol C, Sutawong K, Mayxay M, Rekol H, et al. The spread of artemisinin-resistant Plasmodium falciparum in the Greater Mekong subregion: a molecular epidemiology observational study. The Lancet Infectious diseases. 2017;17(5):491-7.

10. Dondorp AM, Nosten F, Yi P, Das D, Phyo AP, Tarning J, et al. Artemisinin Resistance in Plasmodium falciparum Malaria. N Engl J Med 2009. 2009;361:455-67.

11. Menard D, Fidock DA. Accelerated evolution and spread of multidrug-resistant Plasmodium falciparum takes down the latest first-line antimalarial drug in southeast Asia. The Lancet Infectious diseases. 2019.

12. National Institute of Malariology, Parasitology and Entomology. Guideline for the use of Pyramax in treating patients with malaria infection Hanoi, Vietnam: NIMPE; 2019 [cited 202026 February]. Available from: http://nimpe.vn/userfiles/file/Pyramax/h\%C6\%B0\%E1\%BB\%9Bng\%20d\%E1\%BA\%ABn.pdf.

13. Nsanzabana C, Djalle D, Guérin PJ, Ménard D, González IJ. Tools for surveillance of anti-malarial drug resistance: an assessment of the current landscape. Malaria journal. 2018;17(1):75-.

14. Thriemer K, Hong NV, Rosanas-Urgell A, Phuc BQ, Ha DM, Pockele E, et al. Delayed parasite clearance after treatment with dihydroartemisinin-piperaquine in Plasmodium falciparum malaria patients in central Vietnam. Antimicrob Agents Chemother. 2014;58(12):7049-55. 
15. Brasil P, de Pina Costa A, Pedro RS, da Silveira Bressan C, da Silva S, Tauil PL, et al. Unexpectedly long incubation period of Plasmodium vivax malaria, in the absence of chemoprophylaxis, in patients diagnosed outside the transmission area in Brazil. Malaria Journal. 2011;10(1):122.

16. World Health Organization. Methods for surveillance of antimalarial drug efficacy Geneva, Switzerland: WHO; 2009 [cited 20195 August]. Available from:

https://www.who.int/malaria/publications/atoz/9789241597531/en/.

17. Vietnam Ministry of Health. Guideline for diagnosis and treatment of malaria 2013 [in Vietnamese] Hanoi, Vietnam: Ministry of Health; 2013 [cited 202026 February]. Available from: https://thuvienphapluat.vn/van-ban/the-thao-y-te/Quyet-dinh-3232-QD-BYT-2013-huong-dan-chan-doandieu-tri-benh-Sot-ret-207776.aspx.

18. Vietnam Ministry of Health. Guideline for diagnosis and treatment of malaria 2016 [in Vietnamese] Hanoi, Vietnam: Ministry of Health; 2016 [cited 20195 August]. Available from:

https://thuvienphapluat.vn/van-ban/The-thao-Y-te/Quyet-dinh-4845-QD-BYT-Huong-dan-chan-doan-dieutri-benh-Sot-ret-2016-321533.aspx.

19. World Health Organization. Antimalarial drug efficacy and drug resistance Geneva, Switzerland: WHO; 2018 [cited 202026 February]. Available from:

https://www.who.int/malaria/areas/treatment/drug_efficacy/en/.

20. Huynh HQ. An update on drug-resistant Plasmodium falciparum malaria in Vietnam and worldwide [in Vietnamese] Quy Nhon, Vietnam: Institute of Malariology, Parasitology and Entomology QuyNhon; 2017 [cited 201911 August ]. Available from: http://www.impe-qn.org.vn/impe-qn/vn/portal/InfoDetail.jsp? area $=58 \&$ cat $=1067 \& I D=11105$.

21. Thien HV, Chien VT, Anh TK. Severe malaria in a provincial hospital in Vietnam. Lancet (London, England). 1990;336(8726):1316.

22. Goldlust SM, Thuan PD, Giang DDH, Thang ND, Thwaites GE, Farrar J, et al. The decline of malaria in Vietnam, 1991-2014. Malaria journal. 2018;17(1):226.

23. Kar NP, Kumar A, Singh OP, Carlton JM, Nanda N. A review of malaria transmission dynamics in forest ecosystems. Parasit Vectors. 2014;7:265-.

24. Lindsay SW, Martens WJ. Malaria in the African highlands: past, present and future. Bulletin of the World Health Organization. 1998;76(1):33-45.

25. Bui TC, Markham CM, Ross MW, Williams ML, Beasley RP, Tran LTH, et al. Dimensions of gender relations and reproductive health inequity perceived by female undergraduate students in the Mekong Delta of Vietnam: a qualitative exploration. International Journal for Equity in Health. 2012;11(1):63. 
26. Thanh PV, Van Hong N, Van Van N, Van Malderen C, Obsomer V, Rosanas-Urgell A, et al. Epidemiology of forest malaria in Central Vietnam: the hidden parasite reservoir. Malaria journal. 2015;14:86-.

27. Kitchen AD, Chiodini PL. Malaria and blood transfusion. Vox sanguinis. 2006;90(2):77-84.

28. Alavi SM, Alavi L, Jaafari F. Outbreak investigation of needle sharing-induced malaria, Ahvaz, Iran. International journal of infectious diseases : IJID : official publication of the International Society for Infectious Diseases. 2010;14(3):e240-2.

29. Rogerson SJ. Management of malaria in pregnancy. Indian J Med Res. 2017;146(3):328-33.

30. Muhindo MK, Kakuru A, Jagannathan P, Talisuna A, Osilo E, Orukan F, et al. Early parasite clearance following artemisinin-based combination therapy among Ugandan children with uncomplicated Plasmodium falciparum malaria. Malaria Journal. 2014;13(1):32.

31. World Health Organization. Severe falciparum malaria. World Health Organization, Communicable Diseases Cluster. Transactions of the Royal Society of Tropical Medicine and Hygiene. 2000;94 Suppl 1:S1-90.

32. Camponovo F, Bever CA, Galactionova K, Smith T, Penny MA. Incidence and admission rates for severe malaria and their impact on mortality in Africa. Malaria journal. 2017;16(1):1-.

33. Arévalo-Herrera M, Lopez-Perez M, Medina L, Moreno A, Gutierrez JB, Herrera S. Clinical profile of Plasmodium falciparum and Plasmodium vivax infections in low and unstable malaria transmission settings of Colombia. Malaria Journal. 2015;14(1):154.

34. Saravu K, Rishikesh K, Kamath A. Determinants of mortality, intensive care requirement and prolonged hospitalization in malaria - a tertiary care hospital based cohort study from South-Western India. Malaria journal. 2014;13:370-.

35. Gomes AP, Vitorino RR, Costa AdP, Mendonça EGd, Oliveira MGdA, Siqueira-Batista R. Malária grave por Plasmodium falciparum. Revista Brasileira de Terapia Intensiva. 2011;23:358-69.

36. Hänscheid T. Current strategies to avoid misdiagnosis of malaria. Clin Microbiol Infect. 2003;9(6):497504.

37. Hase R. Diagnostic delay for imported malaria: A case of Plasmodium falciparum malaria misdiagnosed as common cold. J Gen Fam Med. 2017;19(1):27-9.

38. Al Farsi F, Chandwani J, Mahdi AS, Petersen E. Severe imported malaria in an intensive care unit: A case series. IDCases. 2019;17:e00544-e.

39. Asma U-e, Taufiq F, Khan W. Prevalence and clinical manifestations of malaria in Aligarh, India. Korean J Parasitol. 2014;52(6):621-9. 
40. Woodford J, Shanks GD, Griffin P, Chalon S, McCarthy JS. The Dynamics of Liver Function Test Abnormalities after Malaria Infection: A Retrospective Observational Study. The American journal of tropical medicine and hygiene. 2018;98(4):1113-9.

41. Woodford J, Shanks GD, Griffin P, Chalon S, McCarthy JS. The Dynamics of Liver Function Test Abnormalities after Malaria Infection: A Retrospective Observational Study. Am J Trop Med Hyg. 2018;98(4):1113-9.

42. Al Farsi F, Chandwani J, Mahdi AS, Petersen E. Severe imported malaria in an intensive care unit: A case series. IDCases. 2019;17:e00544.

43. Choi IH, Hwang PH, Choi SI, Lee DY, Kim MS. Delayed Diagnosis of Falciparum Malaria with Acute Kidney Injury. J Korean Med Sci. 2016;31(9):1499-502.

44. Trampuz A, Jereb M, Muzlovic I, Prabhu RM. Clinical review: Severe malaria. Crit Care. 2003;7(4):31523.

45. Woodrow CJ, White NJ. The clinical impact of artemisinin resistance in Southeast Asia and the potential for future spread. FEMS microbiology reviews. 2017;41(1):34-48.

46. Commey J, Quarm-Goka B, Agyepong I. Persistent fever in severe malaria in children. The Central African journal of medicine. 1994;40(9):257-60.

47. Ouji M, Augereau JM, Paloque L, Benoit-Vical F. Plasmodium falciparum resistance to artemisininbased combination therapies: A sword of Damocles in the path toward malaria elimination. Parasite. 2018;25:24.

48. Nsanzabana C. Resistance to Artemisinin Combination Therapies (ACTs): Do Not Forget the Partner Drug! Tropical medicine and infectious disease. 2019;4(1).

49. van der Pluijm RW, Imwong M, Chau NH, Hoa NT, Thuy-Nhien NT, Thanh NV, et al. Determinants of dihydroartemisinin-piperaquine treatment failure in Plasmodium falciparum malaria in Cambodia, Thailand, and Vietnam: a prospective clinical, pharmacological, and genetic study. The Lancet Infectious diseases. 2019.

50. Hawkes M, Conroy AL, Kain KC. Spread of Artemisinin Resistance in Malaria. New England Journal of Medicine. 2014;371(20):1944-5.

51. Rasmussen C, Nyunt MM, Ringwald P. Artemisinin-Resistant Plasmodium falciparum in Africa. The New England journal of medicine. 2017;377(3):305-6.

52. Worldwide Antimalarial Resistance Network DPSG. The effect of dosing regimens on the antimalarial efficacy of dihydroartemisinin-piperaquine: a pooled analysis of individual patient data. PLoS Med. 2013;10(12):e1001564-e. 
53. Mahase E. Malaria drugs left ineffective by spread of multidrug resistant parasites in southeast Asia. BMJ. 2019;366:14807.

54. Vietnam Minstry of Health. Guideline for diagnosis and treatment of malaria 2009 [in Vietnamese] Hanoi, Vietnam: Vietnam Ministry of Health; 2009 [cited 20195 Aug]. Available from:

https://vanbanphapluat.co/quyet-dinh-4605-q-c3-90-byt-huong-dan-chan-doan-va-dieu-tri-sot-ret.

55. Roseau JB, Pradines B, Paleiron N, Vedy S, Madamet M, Simon F, et al. Failure of dihydroartemisinin plus piperaquine treatment of falciparum malaria by under-dosing in an overweight patient. Malaria journal. 2016;15:479-.

56. Hodel EM, Kay K, Hayes DJ, Terlouw DJ, Hastings IM. Optimizing the programmatic deployment of the anti-malarials artemether-lumefantrine and dihydroartemisinin-piperaquine using pharmacological modelling. Malaria journal. 2014;13:138-.

57. Moore BR, Batty KT, Andrzejewski C, Jago JD, Page-Sharp M, llett KF. Pharmacokinetics and pharmacodynamics of piperaquine in a murine malaria model. Antimicrob Agents Chemother. 2008;52(1):306-11.

58. Tarning J, Zongo I, Somé FA, Rouamba N, Parikh S, Rosenthal PJ, et al. Population pharmacokinetics and pharmacodynamics of piperaquine in children with uncomplicated falciparum malaria. Clin Pharmacol Ther. 2012;91(3):497-505.

59. Hoglund RM, Workman L, Edstein MD, Thanh NX, Quang NN, Zongo I, et al. Population Pharmacokinetic Properties of Piperaquine in Falciparum Malaria: An Individual Participant Data MetaAnalysis. PLoS Med. 2017;14(1):e1002212-e.

60. Sowunmi A, Adewoye EO, Gbotsho GO, Happi CT, Sijuade A, Folarin OA, et al. Factors contributing to delay in parasite clearance in uncomplicated falciparum malaria in children. Malaria Journal. 2010;9(1):53.

61. Woodrow CJ, White NJ. The clinical impact of artemisinin resistance in Southeast Asia and the potential for future spread. FEMS Microbiol Rev. 2017;41(1):34-48.

\section{Figures}




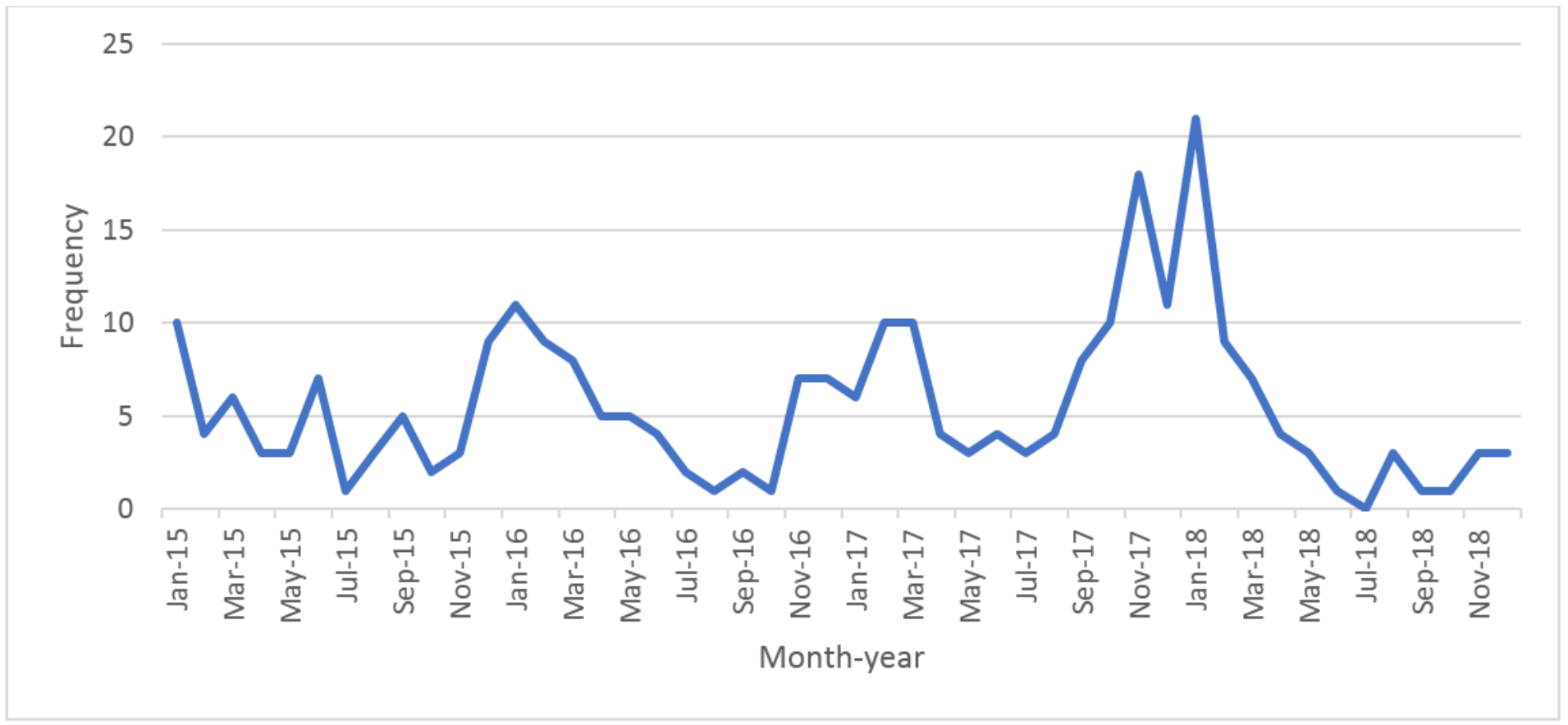

Figure 1

Frequency distribution of P. falciparum infected cases between January 2015 and December 2018 